\title{
Challenges in the Design of the Detector Solenoid for the $\mathrm{Mu} 2 \mathrm{e}$ Experiment
}

\author{
R. Ostojic, R. Coleman, I. Fang, M. Lamm, J. Miller, T. Page, Z. Tang, M. Tartaglia, R. Wands
}

\begin{abstract}
The Mu2e experiment at Fermilab is being designed to measure the rare process of direct muon to electron conversion in the field of a nucleus. The experiment comprises a system of three superconducting solenoids which focus secondary muons from the production target and transport them to the stopping target, while minimizing the associated background. The Detector Solenoid is the last magnet in the transport line and it consists of an axially graded-field section at the upstream end, where the stopping target is located, and a spectrometer section with uniform field at the downstream end for accurate momentum measurement of the conversion elections. The Detector Solenoid has a warm bore of $1.9 \mathrm{~m}$ and is $10.75 \mathrm{~m}$ long. The stored energy of the magnet is $30 \mathrm{MJ}$. The conceptual design of the magnet is presented, in particular the challenge of achieving tight magnetic field specification in a cost-effective design.
\end{abstract}

Index Terms-Detector magnets

\section{INTRODUCTION}

$\mathrm{T}$ HE Mu2e experiment at Fermilab is being designed to measure the rare process of direct muon to electron conversion in the field of a nucleus [1]. The experiment comprises a system of three superconducting solenoids: the Production Solenoid (PS), which houses the production target, the Transport Solenoid (TS) which focuses the secondary muons and transports them to the stopping target, and the Detector Solenoid (DS) where the stopping target, the tracker, calorimeter and other experiment equipment are installed. The magnet system of the Mu2e experiment is described in more detail in ref. [2].

The main functions of the DS are to provide a graded field in the region of the stopping target and to provide a precision magnetic field in a volume large enough to house the tracker downstream of the stopping target. The inner diameter of the magnet cryostat is $1.9 \mathrm{~m}$ and its length is $10.75 \mathrm{~m}$. The inner cryostat wall supports the stopping target, tracker, calorimeter and other equipment installed in the DS. This volume is under vacuum during operation. It is open on the upstream side, where the DS cryostat interfaces with the TS, and sealed on the downstream side by the muon beam stop.

The DS is designed to satisfy the field and operational requirements defined in the DS specification document [3].

Manuscript received October 9, 2012. This work was supported in part by the Fermi Research Alliance under DOE Contract DE-AC02-07CH11359.

R. Ostojic is with CERN, Geneva, Switzerland. Phone: +41-22-767-5146, fax : +41-22-766-7973, e-mail: ranko.ostojic@cern.ch.

R. Coleman, I. Fang, M. Lamm, T. Page, R. Tartaglia and R. Wands are with Fermilab, Batavia, IL 60510, USA.

J. Miller is with Boston University, Boston, MA 02215, USA.
The overall layout of the solenoid is shown in Fig. 1. It consists of two sections: a "gradient section", which is about $4 \mathrm{~m}$ long, and a "spectrometer section" of about $6 \mathrm{~m}$. The magnetic field at the entrance of the gradient section is $2 \mathrm{~T}$ and decreases linearly to $1 \mathrm{~T}$ at the entry of the spectrometer section, where it is uniform over $5 \mathrm{~m}$.

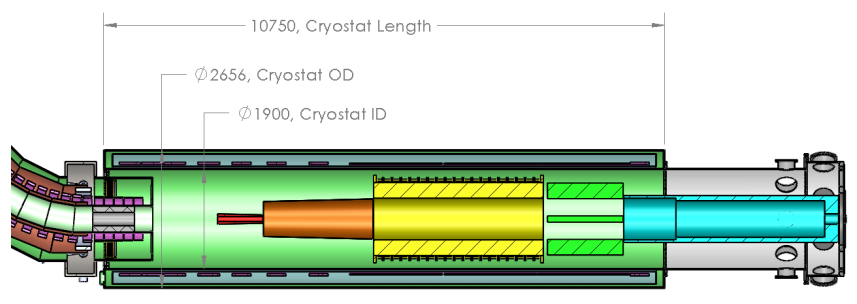

Fig. 1. Layout of the Detector Solenoid coils and cryostat.

The DS coil design is based on a high purity aluminum sheath surrounding a $\mathrm{Nb}$-Ti Rutherford cable. This type of conductor has been used successfully in many similar superconducting detector solenoids [4]. Aluminum has very small resistivity and a large thermal conductivity at low temperatures providing excellent stability. Furthermore, aluminum stabilized conductors can be extruded in lengths of several kilometers. Precise rectangular conductor shapes can be obtained, allowing for high accuracy in the coil winding.

Two types of conductor are required, both $20 \mathrm{~mm}$ high. The "narrow" (5.25 mm wide) conductor is used in the gradient section, while the "wide" ( $7 \mathrm{~mm}$ wide) conductor is used in the spectrometer section. The dimensions are optimized to give the required field when identical current is transported in both conductors. The conductors contain $\mathrm{Nb}$ - Ti Rutherford cables with 12 and 8 strands, respectively. The strands have a diameter of $1.3 \mathrm{~mm}, \mathrm{SC} / \mathrm{Cu}$ ratio of 1.0 and critical current of $2750 \mathrm{~A} / \mathrm{mm} 2(4.2 \mathrm{~K}, 5 \mathrm{~T})$. As a result, the conductors have significant stability and safety margins.

In the baseline design, the gradient section is wound in two layers using the "narrow" conductor $(20 \mathrm{~mm} \times 5.25 \mathrm{~mm})$, which is necessary to obtain a field of $2 \mathrm{~T}$. The field gradient is obtained by introducing several sets of spacers between coil modules. The field uniformity in the spectrometer section is achieved with a "wide" conductor $(20 \mathrm{~mm} \times 7 \mathrm{~mm})$, wound as a single layer coil.

It is envisaged that the DS coil will be wound in standardized modules on accurately machined collapsible mandrels. After curing, the winding mandrels are extracted 
and the outer aluminum support cylinders are placed over each module and the assembly epoxy bonded. The preassembled modules are then electrically connected and bolted together with spacers in a single cold mass before installation in the cryostat.

The DS (cold mass and cryostat) weighs about 39 metric tons. With the stored energy of $30 \mathrm{MJ}$, the ratio of stored energy to mass of the DS magnet is $3.4 \mathrm{~kJ} / \mathrm{kg}$, which places the magnet within the range of conservative designs [5]. The main parameters of the magnet are summarized in Table I.

TABLE I

SUMMARY OF DETECTOR SOLENOID PARAMETERS

\begin{tabular}{lc}
\hline \multicolumn{1}{c}{ Parameter } & \\
\hline Coil inner diameter & $2100 \mathrm{~mm}$ \\
Coil Outer diameter & $2186 \mathrm{~mm}$ \\
Coil length & $10150 \mathrm{~mm}$ \\
Coil mass $(m)$ & $8800 \mathrm{~kg}$ \\
Cryostat inner diameter & $1900 \mathrm{~mm}$ \\
Cryostat outer diameter & $2656 \mathrm{~mm}$ \\
Cryostat length & $10750 \mathrm{~mm}$ \\
Cryostat mass & $30,000 \mathrm{~kg}$ \\
Nominal temperature & $4.5 \mathrm{~K}$ \\
Nominal current & $6115 \mathrm{~A}$ \\
Peak field & $2.2 \mathrm{~T}$ \\
Stored energy $(E)$ & $30 \mathrm{MJ}$ \\
$E / m$ & $3.4 \mathrm{~kJ} / \mathrm{kg}$ \\
\hline
\end{tabular}

\section{MAGNET DESIGN}

\section{A. Coil}

The DS coil, shown in Fig. 2, consists of 11 modules, seven in the gradient section and four in the spectrometer section. Two module types are used in the gradient section. They are wound in two-layers and differ only in the number of turns (that is active length). The modules are separated by premachined spacers to give the required field profile. The spectrometer section contains three identical modules, wound as single layer coils. Finally, to provide a sharp field fall-off, a short two-layer module, identical to that used in the gradient section, is mounted on the downstream end of the magnet.

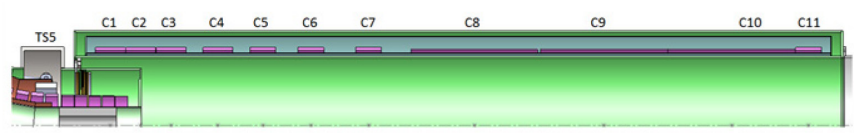

Fig. 2. Layout of the DS coil modules. TS coils are shown on the left.

The two-layer modules are bonded with epoxy to the inner surface of a $2 \mathrm{~cm}$ thick 5083-0 aluminum support cylinder, while the thickness of the cylinder is $1 \mathrm{~cm}$ for single layer modules. These thicknesses are chosen so that the support cylinder can resist the stress generated by the full magnetic pressure. The cylinder also provides a load path to the cold mass support system. This aluminum grade is non-heattreatable and can be reliably welded without loss of strength. The thickness of the support cylinder is locally increased in the downstream end to provide additional stiffness in the region of the axial supports.

The equivalent stress in the coil at various loading stages is shown in Fig. 3. The maximum equivalent stress in the conductor is $39 \mathrm{MPa}$. In the support cylinder, the maximum equivalent stress is $50 \mathrm{MPa}$. Both maxima occur in the first module of the gradient section. The stresses in the major part of the magnet are much lower. The maximum allowable working stress of 5083-0 aluminum at $77 \mathrm{~K}$ is $107 \mathrm{MPa}$, so that the support cylinder provides structural integrity of the coil even in the case when it takes the full load. The maximum equivalent stress in the coil is such that some plastic deformation can be expected during the first powering of the magnet. This is allowable as long as the strain is small [6].

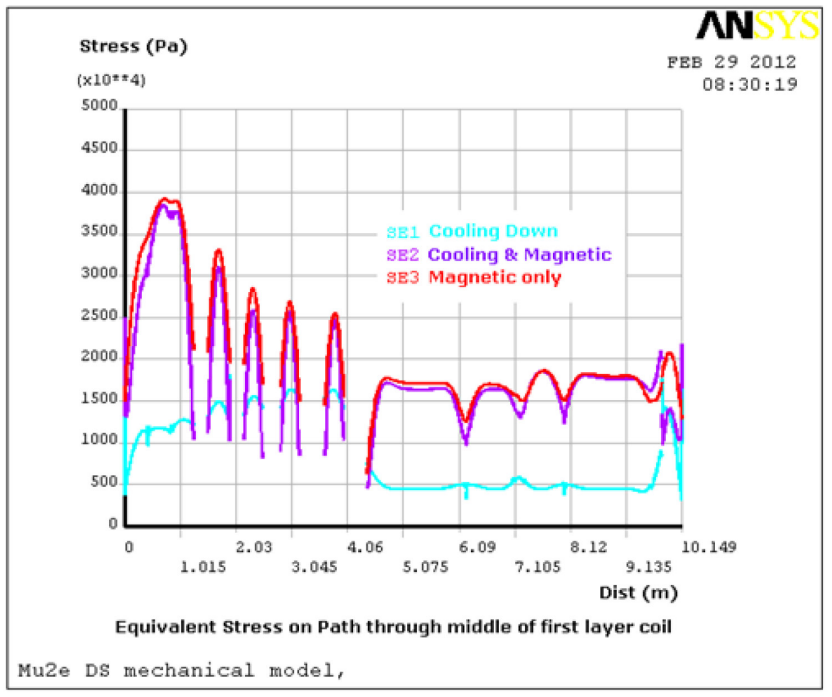

Fig. 3. Equivalent stress inside the first coil layer for the three load cases: cooldown, powering, and combined cooldown and powering.

\section{B. Cryostat}

The DS cold mass is held within the cryostat by radial and axial support systems, shown in Fig. 4. The radial support system consists of 8 pairs of tangentially opposed Inconel 718 rods, 4 pairs at each end. The rods are $66 \mathrm{~cm}$ long, and $12.7 \mathrm{~mm}$ in diameter. The axial support system consists of eight 1.25" Schedule 10 Inconel 718 rods, located on the downstream end only. At the ends of each rod there is a spherical bearing to accommodate motion due to thermal contraction. The warm ends of the axial supports connect directly to the cryostat outer shell.

The support system was dimensioned on the basis of maximum loads (weight and magnetic force). The asymmetry of the DS inherently forces the magnet towards the TS, and the axial support system is always in tension. This axial force depends on how accurately the solenoid is positioned relative to the TS. Axial and radial forces were calculated with a 3-D finite element model that included both the TS and the DS magnets. The assumption was made that the misalignments would not exceed $2 \mathrm{~cm}$. The axial force is at a maximum when the TS is operating and equals 100 tons. The radial centering force with the TS off can reach 1 ton for an installation $2 \mathrm{~cm}$ from the nominally centered position.

The cryostat vessel consists of concentric $2 \mathrm{~cm}$ thick stainless steel cylindrical shells connected by annular end 
rings. The shells are sized according to ASME Section VIII, Div. 1 rules for cylindrical shells under external pressure. Given that the bore of the solenoid may be evacuated while the magnet is warm (at room temperature and pressure), both the inner and outer shells have a design external pressure of 1 atmosphere. In addition, the inner shell must accommodate approximately 10 metric tons of detectors, shielding and other equipment. This load rests on rails attached to the inside of the shell. The cryostat sits on two saddles, positioned very close to the ends of the vessel, also shown in Fig. 4.

The cryostat provides the load path for cold mass reactions through its support system. The axial supports bear directly against the cryostat outer shell, and transmit the forces to the saddle support. This arrangement essentially produces no stresses on the cryostat. The warm ends of the radial supports attach to the cryostat through towers, which transmit the load through the outer shell to the saddles.

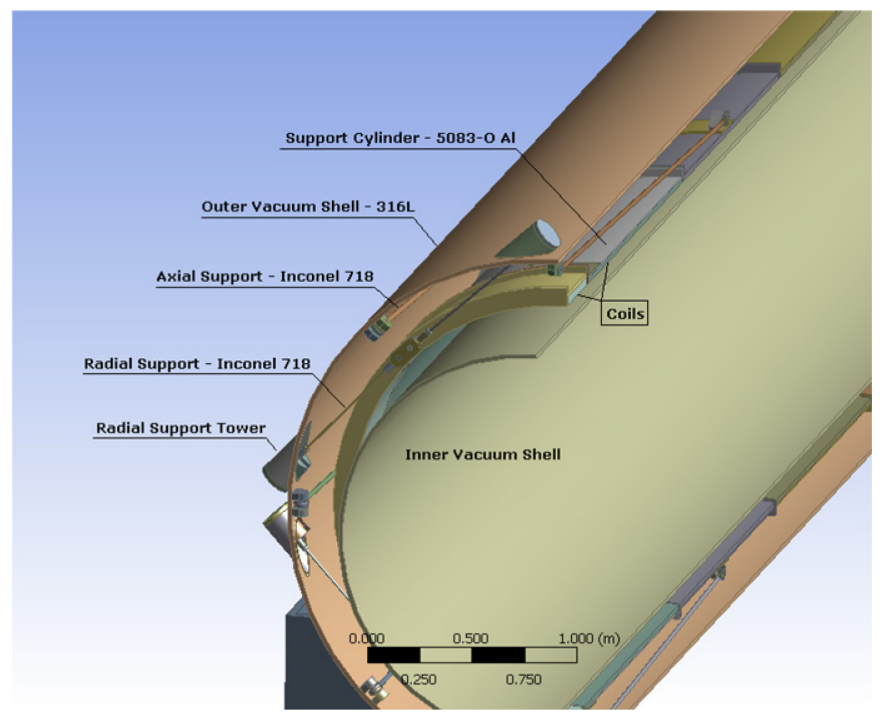

Fig. 4. Radial and axial supports of the cold mass at the downstream end of the DS magnet. The downstream support saddle for the cryostat is also shown.

\section{Quench Protection}

The DS must be protected in case of a quench, whether occurring in the body or in the leads of the magnet, and from any malfunctions of the cooling and powering system. The solenoid will be powered with a dedicated power convertor. The circuit will contain protection elements for the solenoids (circuit breakers and extraction resistors) as well as for ancillary equipment (current leads, bus bars, grounding circuit). The normal powering and discharge of the circuit will be performed with ramp rates that pose negligible risk of quench. In case of minor faults, a slow discharge of the circuit will be launched without provoking a magnet quench. Only in case of a major fault shall the fast discharge be engaged and the magnet energy extracted quickly in a controlled way. This mode of protection relies on extraction resistors, dimensioned such that the peak voltage to ground is less than $500 \mathrm{~V}$.

An analysis of the quench propagation using the QLASA code [7], in the adiabatic regime, indicated that for a wide range of parameters (starting quench positions and different RRR of the Al-stabilizer) the peak temperature in the coil remains below $85 \mathrm{~K}$. As the DS design relies on Al-stabilized conductor and an Al-support cylinder, it is expected that the protection properties of the magnet will be strongly enhanced by quench-back. A simulation model based on ANSYS was developed to study behavior during quench in this case [8]. The model includes all material and dimensional features of the conductors, and the field and eddy current distribution in the coil and its support cylinder as function of time. This model shows that without quench-back, the maximum temperature in the coil is $80 \mathrm{~K}$, very close to the adiabatic result. In case of a full model including quench-back, initiated by discharging the magnet through a dump resistor, all sections of the magnet are resistive within a few seconds after the quench begins. The maximum temperature in the coil is in this case $30 \mathrm{~K}$. These results indicate that the DS magnet can be safely protected whatever the origin of the quench.

\section{Thermal Analysis}

The DS coil is indirectly cooled by liquid helium flowing in tubes welded to the outer surface of the support cylinders. The liquid helium will be supplied by either a forced-flow or thermosiphon system. In the case of forced-flow cooling a mass flow rate of $50 \mathrm{~g} / \mathrm{s}$ and a pressure of $0.23 \mathrm{MPa}$ are provided by the Mu2e cryogenic system. Given the expected heat load of $20 \mathrm{~W}$ at $4.5 \mathrm{~K}$ from mechanical supports and radiation, the temperature of the liquid helium leaving the magnet will be 0.1 degree above the entry temperature. The maximum coil temperature rise is then $0.49 \mathrm{~K}$ and occurs in the region of the axial supports.

\section{MAGNETIC FIELD ANALYSIS}

For the purpose of field analysis, the DS can be divided into four sections: DS1-DS4, shown in Fig. 5. The field on the axis of the magnet is also shown in the figure. As can be seen, there is a significant contribution of the TS in the gradient section (DS1) which must be accounted for in the DS design. Conversely, the DS coils C1-C3 have a large impact on the field quality in the TS. Using an iterative procedure, the coils of the DS and TS could be optimized to satisfy the field requirements while minimizing the length of the magnets and the volume of superconductor.

The peak field in the DS coils is $2.2 \mathrm{~T}$ and occurs in the first module of the DS1 section. At the operating current of $6.1 \mathrm{kA}$, the magnet is at $45 \%$ of conductor quench current, with a temperature margin of $2.5 \mathrm{~K}$ with respect to the assumed conductor temperature of $5.0 \mathrm{~K}$. The field in other sections is lower with a consequently larger temperature margin.

The precision of the magnetic field in the DS1 and DS3DS4 sections are the major design and fabrication challenges for the DS magnet. The nominal on- and off-axis field profiles in the DS1 and DS3-DS4 sections are shown respectively in Fig. 6 and Fig. 7. The allowed field uniformity requirements are also shown. The field uniformity requirements are most stringent in the DS3 section $( \pm 1 \%)$, and are somewhat relaxed in the DS1 and DS4 sections ( $\pm 5 \%)$. 


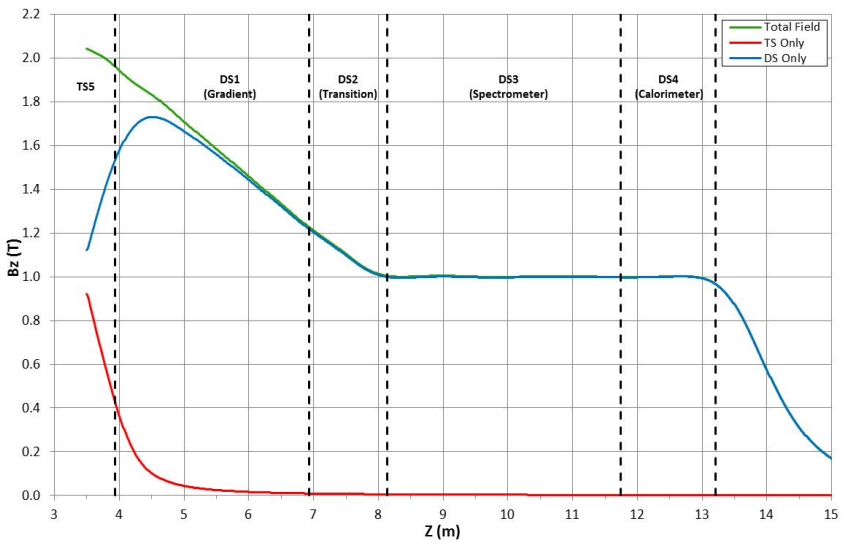

Fig. 5. Axial field on the axis of the DS magnet. There is an important contribution of the TS magnet in the gradient section (DS1).

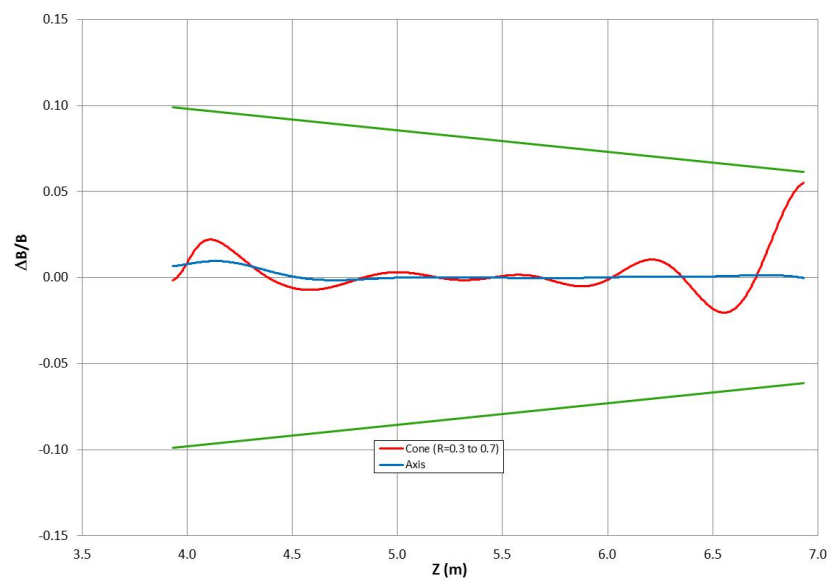

Fig. 6. Comparison of the magnetic field with field tolerance in the gradient section (DS1). Field tolerance is shown in green. $\Delta \mathrm{B}$ is relative to a uniform gradient of $-0.25 \mathrm{~T} / \mathrm{m}$ and a field of $1.5 \mathrm{~T}$ at the stopping target. The relative field errors $\Delta \mathrm{B} / \mathrm{B}$ on axis (blue), and on a radial cone from $0.3 \mathrm{~m}$ to $0.7 \mathrm{~m}$ starting at the upstream end of DS1 section (red), are shown.

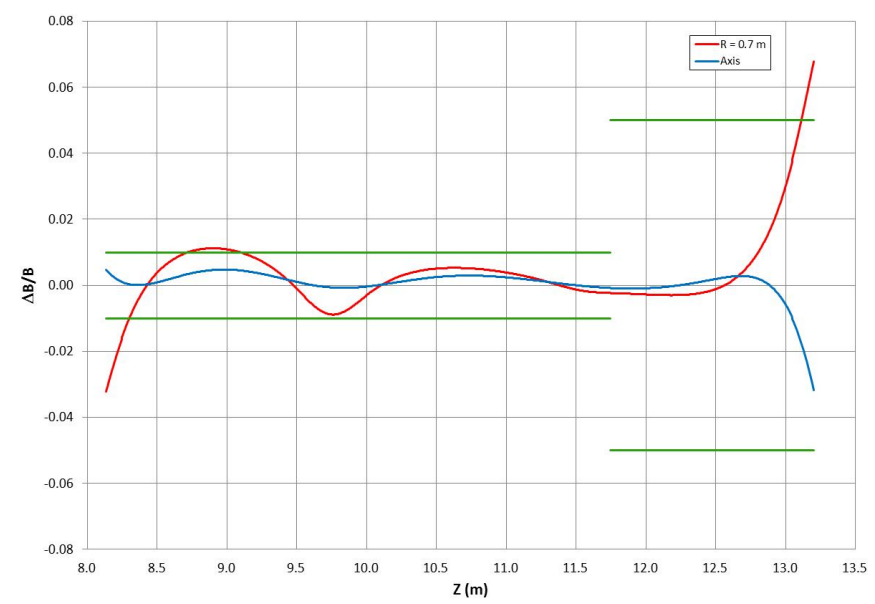

Fig. 7. Comparison of the magnetic field with field tolerance in the spectrometer section (DS3-DS4). Field tolerance is shown in green. $\Delta \mathrm{B}$ is relative to a uniform field of $1.0 \mathrm{~T}$. The relative field errors $\Delta \mathrm{B} / \mathrm{B}$ on axis (blue), and at a radial distance of $0.7 \mathrm{~m}$ (red), are shown.

Detailed sensitivity analysis has established that the length of the coil modules is the most critical for field uniformity. A somewhat lesser sensitivity is due to the relative axial position of the coils, while the transverse geometry of the coils after assembly of the support cylinder and final curing (coil radius and ovality) are the least important. A full tolerance study was performed, assuming random choice of geometric parameters of each of the eleven coil modules. The tolerance intervals assumed corresponded to the standards encountered in precision machining. The so-obtained field distributions were analyzed for a large number of cases. The most probable field distributions in the gradient and spectrometer sections, as well as the peak field deviations, were shown to be in all cases within the field uniformity requirements.

The Mu2e experiment is very much dependent on minimizing the background, and any deviation of the field must be closely analyzed by sophisticated and time consuming tracking using the full 3D field. To retain the possibility of correcting the field once the conductors and coil modules are in production, we are considering several possibilities for local and global adjustment of the field profile in the DS3-DS4 sections. A promising technique is to modify the spacers between modules C6-C8 and C10-C11 (Fig. 2), adjusting their relative axial position. Other possibilities include custommade modules $\mathrm{C} 8$ and $\mathrm{C} 11$. The favored trimming technique will be decided upon once the consequences of local field deviations on the experimental background are fully understood.

\section{CONCLUSION}

The Detector Solenoid for the Mu2e experiment at Fermilab is a $2 \mathrm{~T}$ magnet with a conservative $E / m$ ratio. However, the magnet is large and the main challenge in its design and construction is achieving tight magnetic field uniformity specification in a cost-effective way. The conceptual design of the magnet is complete and procurement of test samples of the superconducting cable and its pure-Al sheath are on order.

\section{REFERENCES}

[1] R. Tschirhart, "The Mu2e experiment at Fermilab", Nucl.Phys.Proc.Suppl. 210-211 (2011) 245-248.

[2] M.J. Lamm et al., "Solenoid Magnet System for the Fermilab Mu2e Experiment", IEEE Trans. Appl. Supercond. Vol. 22, submitted for publication.

[3] Mu2e Conceptual Design Report (CDR) R. Ray et al. see: http://mu2e.fnal.gov/public/hep/general/proposals.shtml

[4] H. Minemura et al., "Construction and testing of a $3 \mathrm{~m}$ diameter x $5 \mathrm{~m}$ superconducting solenoid for the Fermilab collider detector facility (CDF) ”, Nucl. Instrum. Methods A, vol. 238, pp. 18-34, July 1985.

[5] A. Yamamoto, T. Taylor, Y. Makida, K. Tanaka, "Next Step in the Evolution of Superconducting Detector Magnets", IEEE Trans. Appl. Supercond., vol. 18, No. 2, pp. 362-366, June 2008.

[6] B. Blau et al., "The CMS Conductor", IEEE Trans. Appl. Supercond., vol. 12, No. 1, pp. 345-348, March 2002.

[7] L. Rossi, M. Sorbi, "QLASA: a computer code for quench simulation in adiabatic multicoil superconducting windings," INFN/TC-04/13, July 2004.

[8] M. Wake et al., "Complete Quench Simulation of Large Soleniod Magnet", IEEE Trans. Appl. Supercond. Vol. 22, submitted for publication. 